\title{
Risk factors of recurrent erysipelas in adult Chinese patients: a prospective cohort study
}

\author{
Ang $\mathrm{Li}^{1^{*}}$, Ni Wang ${ }^{2}$, Lingzhi Ge ${ }^{3}$, Hongyan $\mathrm{Xin}^{4}$ and Wenfei Li ${ }^{2}$
}

\begin{abstract}
Background: Erysipelas is a common skin infection that is prone to recur. Recurrent erysipelas has a severe effect on the quality of life of patients. The present study aimed to investigate the risk factors of recurrent erysipelas in adult Chinese patients.

Methods: A total of 428 Chinese patients with erysipelas who met the inclusion criteria were studied. The patients were divided into the nonrecurrent erysipelas group and the recurrent erysipelas group. Clinical data were collected on the first episode and relapse of erysipelas. The patients were followed up every 3 months. Statistical analysis was performed to analyze and determine the risk factors of erysipelas relapse.

Results: Univariate analysis was performed to analyze the data, including surgery, types of antibiotics administered in the first episode, obesity, diabetes mellitus, venous insufficiency, lymphedema, and malignancy. The differences between the groups were statistically significant $(p<0.05)$. The Cox proportional hazards regression model analysis showed that the final risk factors included surgery, obesity, diabetes mellitus, venous insufficiency, and lymphedema.

Conclusions: Surgery, obesity, diabetes mellitus, venous insufficiency, and lymphedema are considered as risk factors for recurrent erysipelas.
\end{abstract}

Keywords: Erysipelas, Recurrent, Risk factor, Prospective, Cohort study

\section{Background}

Erysipelas is an acute inflammation of the skin and its reticular lymphatic vessels, which is prone to recur in the lower extremities, face, and other anatomical areas. It is frequently caused by $\beta$-hemolytic streptococci group A (Streptococcus pyogenes) and rarely caused by streptococci groups B, C, or G $[1,2]$. The following characteristics differentiate erysipelas from other forms of tissue infections: (1) an acute, warm, slightly painful, bright red erythema with a shiny surface and a sharply defined margin as well as tongue-shaped processes, usually

\footnotetext{
* Correspondence: liang_1@sjtu.edu.cn

'Department of Orthopaedic Surgery, Shanghai Jiao Tong University Affiliated Sixth People's Hospital, 600 Yishan Road, Shanghai 200233, China Full list of author information is available at the end of the article
}

located a few centimeters away from the entry site; (2) a systemic inflammatory response immediately from the onset, marked by fever or at least shivering and rarely chills; and (3) increased erythrocyte sedimentation rate (ESR), increased C-reactive protein (CRP) level, and/or neutrophilia [3].

Acute and recurrent erysipelas can be differentially diagnosed on the basis of the following aspects: First, if the disease recurs in the original site, then it is termed as recurrent erysipelas; however, recurrence is not considered if two episodes of erysipelas occur in different anatomical areas in the same patient. Second, a patient with recurrent erysipelas could recover naturally in a few days, but the disease could recur within the next weeks or even years after recovery. Third, the duration 
of recurrent erysipelas is longer than that of acute erysipelas. Therefore, it is very important to take preventive measures to avoid the recurrence of erysipelas.

Some risk factors might be related to the recurrence of erysipelas [4]. The present article discusses the risk factors of recurrent erysipelas and provides the theoretical basis for preventing the recurrence of erysipelas.

\section{Methods}

The present study included a prospective cohort of Chinese patients suffering from the first episode of erysipelas. The study patients were mostly residents of Shandong province, and a few patients were Chinese residents from other provinces and cities in China. A total of 525 patients with erysipelas were hospitalized in our hospital from June 2014 to June 2019, and a total of 428 inpatients with erysipelas who met the inclusion criteria were studied. The study was formally approved by the ethics committee of the First Affiliated Hospital of Shandong First Medical University.

Because $\beta$-hemolytic streptococci are sensitive to penicillin, many patients with erysipelas were administered penicillin for 7-14 days. If the patient was allergic to penicillin, clindamycin or clarithromycin or moxifloxacin was administered [3]. Other useful treatment measures included keeping the infected area elevated, placing cooling packs on the infected skin area, and applying a prescribed cream on the lesion. When the signs and symptoms subsided and laboratory biochemical markers recovered to normal after treatment, erysipelas was cured. The cohort was followed up every 3 months after being cured and discharged to observe the presence of recurrent erysipelas. All clinical data were obtained through interview, examinations, medical record, home visit, and telephone inquiry.

The diagnosis of erysipelas was based on the signs, symptoms, and results of laboratory biochemical markers consistent with the characteristics of erysipelas. The diagnosis of recurrent erysipelas was based not only on the signs, symptoms, and results of laboratory biochemical markers of erysipelas, but also on the lesions occurring at the same anatomical site as the primary erysipelas.

The patients were divided into the nonrecurrent erysipelas (NRE) group and the recurrent erysipelas (RE) group. NRE was defined as no recurrence of erysipelas, and RE was defined as one or more episodes of erysipelas during follow-up. All patients met the following inclusion criteria: (i) diagnosed to have erysipelas, (ii) over 18 years of age, (iii) no history of previous episodes of erysipelas, (iv) no other skin and soft tissue infections, (v) availability of complete information of risk factors influencing recurrent erysipelas, and (vi) available for follow-up every 3 months after being cured and discharged.
The following potential risk factors for erysipelas relapse were identified and investigated in the present study: age, sex, seasons, anatomical areas, clinical types (vulgaris type and other types), systemic symptoms, disruption of skin barrier (tinea pedis, other dermatoses, and insect bites), surgery, types of antibiotics administered, obesity $(\mathrm{BMI} \geq 28)$ [5], diabetes mellitus, venous insufficiency, lymphedema, cardiovascular diseases, cerebrovascular diseases, lung diseases, liver and kidney diseases, malignancy, and autoimmune diseases. For insect bite reaction, it is manifested as papules, edematous erythema, or blisters on the bite. A small bump frequently accompanied with itching and redness develop on the skin after insect bite. Uric acid arthritis manifests as hyperuricemia. The affected joint shows severe pain, and the first joint often involves the first metatarsophalangeal joint. The characteristics of the above-mentioned diseases can be distinguished from those of primary erysipelas. In addition, stasis dermatitis is a chronic skin disease of the lower limbs that manifests as scaly, itchy skin with lymphedema, which often leads to dark brown skin pigmentation. It is caused by varicose veins. Recurrent erysipelas can cause lymphatic drainage disorder, resulting in the accumulation of lymphatic fluid in the subcutaneous tissues and formation of lymphedema. From the above-mentioned description, these diseases have different mechanisms for forming lymphedema.

\section{Statistical analyses}

Univariate analysis was performed to analyze the differences between the two groups and to determine the factors influencing erysipelas relapse. The data were analyzed with GraphPad Prism ${ }^{\circ}$ version 8 software, and statistical significance was considered at $\alpha=0.05$. We used the ANOVA test and the $\chi^{2}$ or Yates' continuity corrected $X^{2}$ test, when appropriate. Variables were selected as candidates in multivariate analysis with Cox proportional hazards regression model based on the level of significance in univariate analysis. The statistical cutoff point for entry and exclusion was 0.2 and 0.10 , respectively. Multivariate analysis was performed by $\mathrm{IBM}^{\circ}$ SPSS $^{\circ}$ Statistics version 25.

\section{Results}

A total of 428 patients met the study inclusion criteria. During the follow-up, erysipelas did not recur in 359 patients and one or more episodes of erysipelas recurred in 69 patients (Fig. 1). The total number of primary and recurrent episodes was 512 in 428 patients, and 6 episodes were the greatest number of episodes in recurrent patients. The patients enrolled in this study included 231 males (54.0\%) and 197 females (46.0\%). The age range of the patients was 18 to 93 years. The average age of the NRE and RE groups was $57.57 \pm 17.55$ and $63.12 \pm 15.57$ 


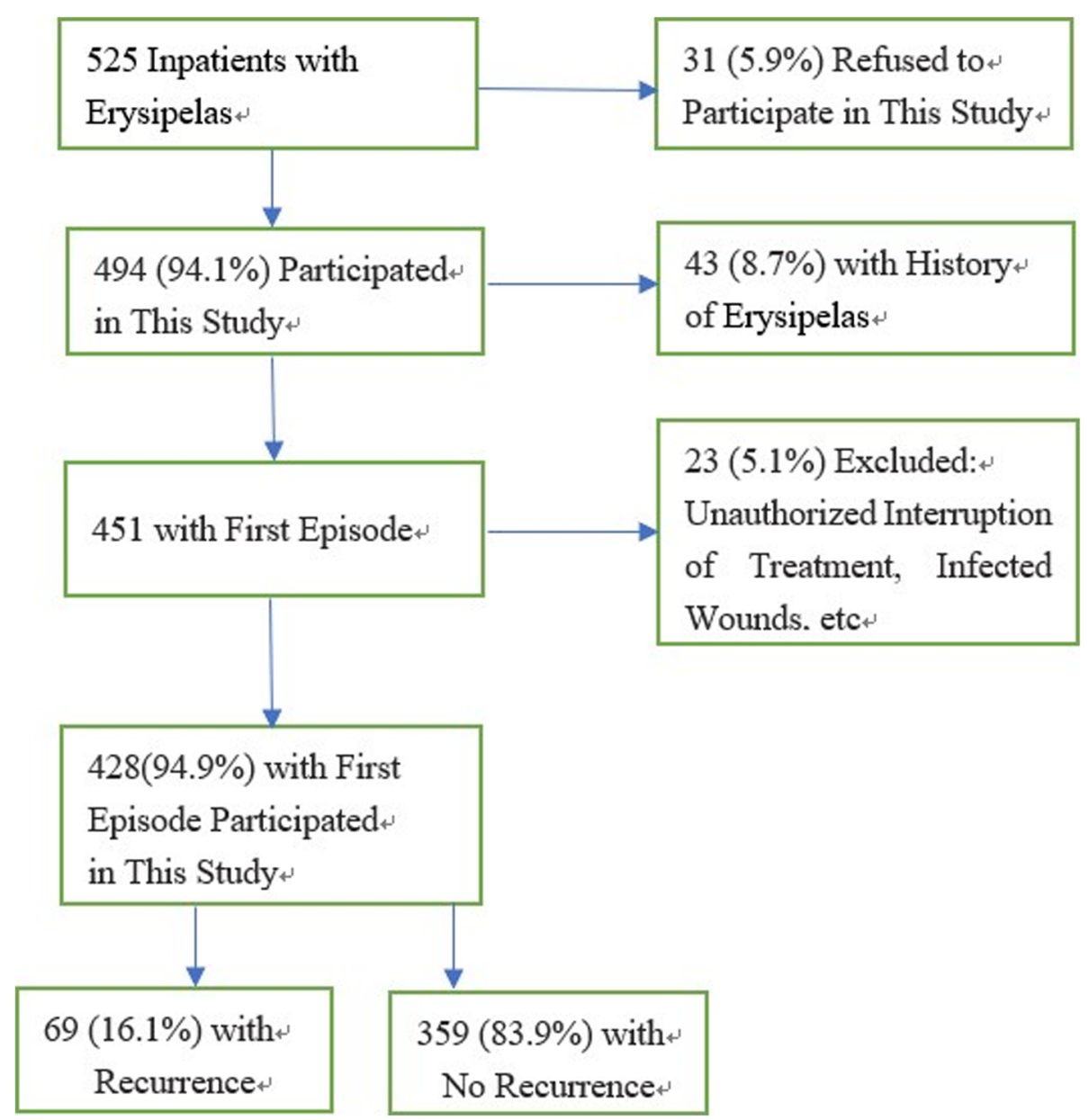

Fig. 1 Study flowchart of risk factors for recurrence of adult inpatients with erysipelas

years, respectively, and the difference in the average age between the two groups was statistically significant $(p<$ 0.05).

Erysipelas was observed in the lower limbs (269 patients $(62.85 \%)$ ), followed by head/face (69 patients $(16.12 \%))$, upper limbs (54 patients (12.62\%)), and trunk (36 patients $(8.41 \%)$ ), respectively. Most of the episodes occurred in the lower limbs (337 episodes), followed by head/face (77 episodes), upper limbs (60 episodes), and trunk (38 episodes). The season could be an initial factor for the development of recurrent erysipelas. Among the four seasons of the year, 133 of 337 episodes occurred in summer. Figure 2 shows the seasonal distribution of episodes of erysipelas in different anatomical areas.

Table 1 shows the results of univariate analysis of demographic and local risk factors. No significant differences were observed regarding gender, clinical types, systemic symptoms, and disruption of skin barrier; however, significant differences were observed regarding surgery and types of antibiotics administered in the first episode between the two groups. Univariate analysis of general risk factors related to erysipelas relapse showed that cardiovascular diseases, cerebrovascular diseases, lung diseases, liver and kidney diseases, and autoimmune diseases were not significant risk factors $(p>0.05)$. As shown in Table 2, the differences in obesity, diabetes mellitus, venous insufficiency, lymphedema, and malignancy were statistically significant $(p<0.05)$.

The Cox proportional hazards regression model was used to analyze the risk factors selected from univariate analysis. The analysis showed that surgery, obesity, diabetes mellitus, venous insufficiency, and lymphedema were risk factors for the recurrence of erysipelas (Table 3).

\section{Discussion}

Erysipelas is a bacterial infectious disease with a tendency to recur. Its recurrence may lead to skin damage, scars, and chronic lymphedema, eventually resulting in the development of elephantiasis, which poses a risk to people's health and increases medical costs and social burden [6]. Therefore, it is crucial to understand the risk 


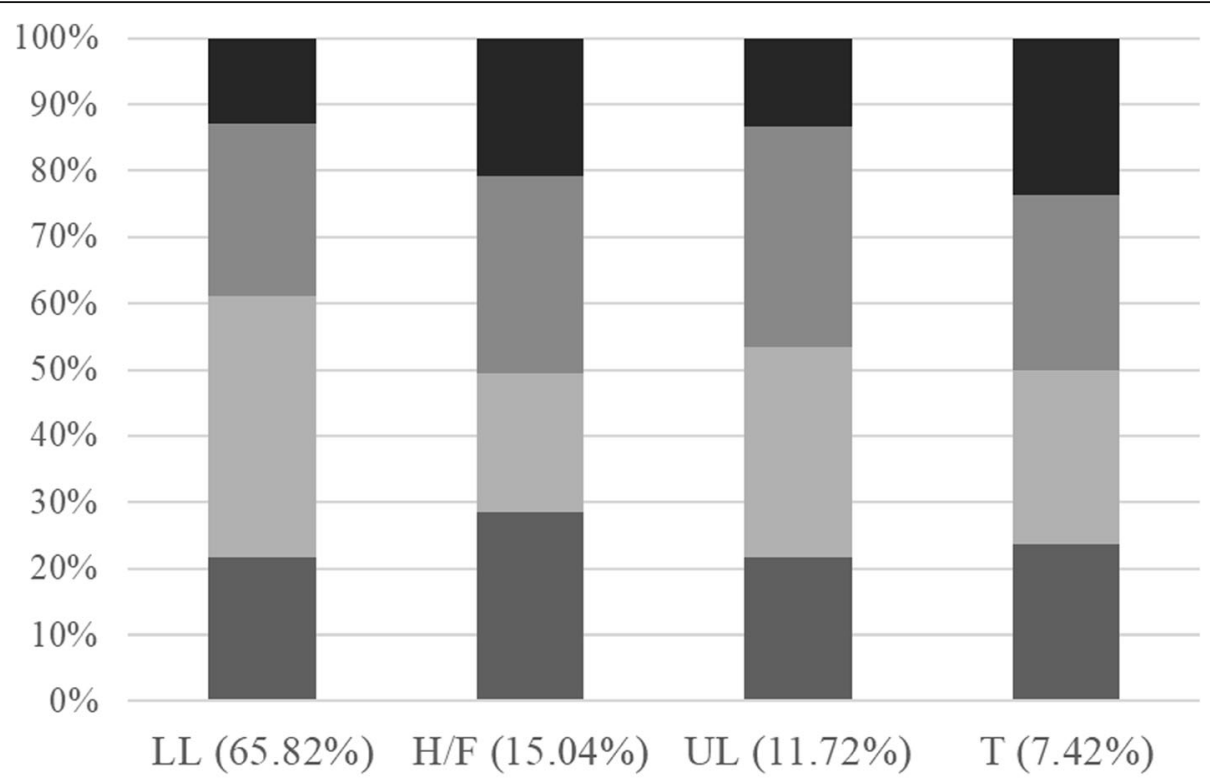

$\square$ Spring $\square$ Summer $\square$ Autumn $\square$ Winter

Fig. 2 The seasons' distribution of the first and recurrent episodes in different anatomical area. Abbreviations: $L L$, lower limbs; $H / F$, head and face; UL, upper limbs; T, trunk. Erysipelas was in lower limbs in 269 of 428 patients. Of all the episodes $(n=512)$, most episodes were in LL (65.82\%), followed H/F (15.04\%), UL (11.72\%), T (7.42\%). During the four seasons of the year, 133 (39.47\%) of 337 episodes occurred in summer

Table 1 Univariate analysis of demographic and local risk factors characteristics in the NRE group and RE group

\begin{tabular}{|c|c|c|c|c|c|}
\hline Variable & $\begin{array}{l}\text { NRE } \\
(n=359)\end{array}$ & $\begin{array}{l}\mathrm{RE} \\
(n=69)\end{array}$ & $x^{2}$ & $p$ value & OR $(95 \% \mathrm{Cl})$ \\
\hline Age & $57.57 \pm 17.55$ & $63.12 \pm 15.57$ & - & 0.02 & - \\
\hline Gender & & & 0.215 & 0.64 & $1.1(0.7-1.9)$ \\
\hline male & $167(46.5)$ & $30(43.5)$ & & & \\
\hline female & $192(53.5)$ & $39(56.5)$ & & & \\
\hline Anatomical areas & & & 9.01 & 0.03 & \\
\hline lower limbs & $215(59.9)$ & $54(78.3)$ & & & 1 (reference) \\
\hline head/face & $61(17.0)$ & $8(11.6)$ & & & $0.5(0.2-1.2)$ \\
\hline upper limbs & 49 13.6) & $5(7.2)$ & & & $0.4(0.2-1.0)$ \\
\hline trunk & $34(9.5)$ & $2(2.9)$ & & & $0.2(0.1-0.9)$ \\
\hline Clinical types & & & 0.005 & 0.94 & $0.9(0.3-2.8)$ \\
\hline vulgaris type & $341(95.0)$ & $66(95.7)$ & & & \\
\hline other types & $18(5.0)$ & $3(4.3)$ & & & \\
\hline Systemic symptoms & $147(40.9)$ & $28(40.6)$ & 0.003 & 0.95 & $1.0(0.6-1.7)$ \\
\hline Disruption of Skin barrier & $45(12.5)$ & $11(15.9)$ & 0.591 & 0.44 & $1.3(0.6-2.7)$ \\
\hline Surgery & $62(17.3)$ & $32(46.4)$ & 28.61 & $<0.0001$ & $4.1(2.4-7.1)$ \\
\hline Antibiotic varieties for first episode & & & 28.04 & $<0.0001$ & $5.0(2.6-9.3)$ \\
\hline penicillin & $330(91.9)$ & $48(69.6)$ & & & \\
\hline other classes of antibiotics & $29(8.1)$ & $21(30.4)$ & & & \\
\hline
\end{tabular}


Table 2 Univariate analysis of comorbidities - general risk factors in the NRE group and RE group

\begin{tabular}{|c|c|c|c|c|c|}
\hline Variable & $\begin{array}{l}\text { NRE } \\
(n=359)\end{array}$ & $\begin{array}{l}\mathrm{RE} \\
(n=69)\end{array}$ & $x^{2}$ & $p$ value & OR $(95 \% \mathrm{Cl})$ \\
\hline Obesity $^{a}$ & $62(17.3)$ & $28(40.6)$ & 18.94 & $<0.0001$ & $3.3(1.9-5.7)$ \\
\hline Diabetes mellitus ${ }^{\mathrm{b}}$ & $47(13.1)$ & $16(23.2)$ & 4.70 & 0.03 & $2.0(1.0-3.8)$ \\
\hline Cardiovascular diseases & $86(24.0)$ & $15(21.7)$ & 0.16 & 0.69 & $0.9(0.5-1.7)$ \\
\hline Cerebrovascular diseases & $48(13.4)$ & $10(14.5)$ & 0.06 & 0.80 & $1.1(0.5-2.3)$ \\
\hline Lung diseases & $37(10.3)$ & $8(11.6)$ & 0.10 & 0.75 & $1.1(0.5-2.5)$ \\
\hline Liver and kidney diseases & $34(9.47)$ & $7(10.1)$ & 0.03 & 0.86 & $1.1(0.4-2.5)$ \\
\hline Venous insufficiency & $20(5.6)$ & $16(23.2)$ & 23.32 & $<0.0001$ & $5.1(2.5-10.7)$ \\
\hline Lymphedema & $21(5.85)$ & $18(26.1)$ & 28.62 & $<0.0001$ & $5.7(2.7-11.4)$ \\
\hline Malignancy & $20(5.57)$ & $9(13.0)$ & 5.12 & 0.02 & $2.5(1.1-5.8)$ \\
\hline Autoimmune diseases & $8(2.2)$ & $3(4.3)$ & 1.04 & 0.31 & $2.0(0.6-6.7)$ \\
\hline
\end{tabular}

abesity, body mass index $\geq 28$, ${ }^{\mathrm{b}}$ Diabetes mellitus, Type 2, expect for one patient with type 1

factors for erysipelas recurrence in order to control and prevent it. However, to the best of our knowledge, few studies have been conducted on the risk factors for the recurrence of erysipelas. The present study is the first to report the risk factors for the recurrence of erysipelas in adult Chinese patients in English literature.

Several studies have suggested that erysipelas has a high recurrence rate and that the recurrence of erysipelas could be related to some potential risk factors [7]. The recurrence rate in the present study was $16.12 \%$, which was lower than that reported in a previous study [6]. The reason for this difference may be related to differences in race, living environment, etc. Moreover, the criteria used for erysipelas have not always been so clearly defined in previous case series or other studies so that some of them may have encompassed also S.aureusmediated soft tissue infections (sometimes referred to as cellulitis) which have less tendency to relapse. However, the new diagnostic criteria for erysipelas will have a significant impact on the distinction between erysipelas and cellulitis, and the recurrence rate of erysipelas [3].

The average age of the patients in the present study was relatively old, suggesting that many patients with erysipelas were elderly people. They might be less resistant to infections and had more complications, which were the risk factors for the occurrence and recurrence of

Table 3 Cox proportional hazards regression model analysis of the correlation between erysipelas recurrence and risk factors

\begin{tabular}{llll}
\hline Variable & $\boldsymbol{P}$ value & HR & $\mathbf{9 5 \% C l}$ \\
\hline surgery & $<0.0001$ & 3.527 & $2.410-5.162$ \\
obesity & $<0.0001$ & 27.298 & $16.543-24.044$ \\
diabetes mellitus & $<0.0001$ & 7.693 & $5.153-11.487$ \\
Venous insufficiency & $<0.0001$ & 4.020 & $2.473-6.534$ \\
lymphedema & $<0.0001$ & 3.821 & $2.324-6.283$ \\
\hline
\end{tabular}

Abbreviations: $\mathrm{HR}$ hazard ratio, $\mathrm{Cl}$ confidence interval erysipelas. Hence, older people show more willingness than young people to be hospitalized for treating erysipelas [8].

In the present study, erysipelas was observed in lower limbs in 269 patients $\left(X^{2}=9.01, p=0.03\right.$ vs. other anatomical areas). Of the 512 episodes, most episodes $(65.82 \%)$ occurred in the lower limbs. Furthermore, of the 337 episodes of erysipelas that occurred in the lower limbs, 133 episodes (39.47\%) occurred in summer. Therefore, summer was the season of high incidence of erysipelas.

Univariate analysis showed no significant differences in sex, clinical types, systemic symptoms, and skin barrier disruption between the two groups. These results indicated that these factors were not closely associated with the recurrence of erysipelas. Tinea pedis, dermatoses, and insect bites can disrupt the skin barrier and cause skin infections; hence, the incidence of erysipelas in the lower limbs is higher, especially in summer [9]. However, because these factors caused mild and temporary damage of the skin barrier, they did not seem to be the risk factors for erysipelas relapse [10]. Surgery can change the local tissue structure and cause poor lymphatic reflux $[p<0.0001$, OR 4.1 (95\% CI 2.4-7.1)] [11]. The results of univariate analysis further indicated that surgery was not the only risk factor for erysipelas, but there were other risk factors as well. We also found that the types of antibiotics administered in the first episode were related to erysipelas recurrence $[p<$ 0.0001, OR 5.0 (95\% CI 2.6-9.3)]. The recurrence rate after penicillin administration was lower than that after the administration of macrolides, quinolones, and aminoglycosides. The reason for this difference may be that most of the pathogens that cause erysipelas were more sensitive to penicillin and that the clearance rate of penicillin was higher; hence, the clinical effect was better [12]. 
Univariate analysis showed no significant differences in the incidence of cardio-cerebral-vascular diseases, lung diseases, and liver and kidney diseases between the two groups; this finding suggested that these factors were not directly related to the recurrence of erysipelas. Obesity not only affects blood pressure and venous circulation in the lower limbs, but it also causes insulin resistance, which might lead to diabetes. A sustained increase in blood glucose concentration can cause dysfunction of mononuclear cells, reduce the body's resistance to pathogens, and thus increase the susceptibility of the patient to easily develop infection [13]. Therefore, obesity $[p<0.0001$, OR: 3.3 (95\% CI: $1.9-5.7)]$ and diabetes $[P=0.03$, OR: 2.0 (95\% CI: $1.0-3.8$ ) $]$ can increase the probability of developing infection and lead to the recurrence of erysipelas. Our present study confirmed that malignancy $[p=0.02$, OR: 2.5 (95\% CI:1.1-5.8)], venous insufficiency $[p<0.0001$, OR: 5.1 (95\% CI: $2.5-$ 10.7)], and lymphedema $[p<0.0001$, OR: 5.7 (95\% CI: 2.7-11.4)] were the possible risk factors for the recurrence of erysipelas. These factors sometimes existed independently, but they often interacted and influenced each other. Malignancy may cause patients to develop abnormal immune functions, while malignant radiotherapy may reduce leukocyte count [14]. Surgical treatment can disrupt the barrier function of the skin and affect the lymphatic circulation. For example, radical mastectomy can cause lymphedema in the surgical area [15]. Venous insufficiency can cause lower extremity edema, stasis dermatitis [16], and even ulcers. These conditions might be the risk factors for the occurrence and recurrence of erysipelas. We also analyzed these risk factors by using the Cox proportional hazards regression model. The analysis revealed that surgery, obesity, diabetes mellitus, venous insufficiency, and lymphedema were the probable risk factors for the recurrence of erysipelas.

The present study has some limitations. First, the average length of hospital stay was $12.66 \pm 4.89$ days, and it was longer than that noted in previous studies $[4,17]$. This might be related to the medical insurance system in mainland China; in this system, a medical insurance company pays a certain percentage of medical expenses incurred only when a patient is hospitalized. Second, the C-reactive protein (CRP) level and peripheral blood white blood cell (WBC) count were not investigated. This is because only one previous study showed differences in CRP and WBC between patients with erysipelas and those with recurrent erysipelas [18], but other studies showed no such differences $[12,19]$. In addition, a new study has elaborated the usefulness of CRP and differential (neutrophil) blood count for differentiating between erysipelas and S.aureus-mediated cellulitis [20]. Therefore, we will focus more on this aspect in our future studies. Third, antibiotics produced by different manufacturers may affect the clinical outcomes observed in the present study.

\section{Conclusion}

Erysipelas is an infectious skin disease that frequently occurs in elderly people. It usually tends to recur, and surgery, obesity, diabetes mellitus, venous insufficiency, and lymphedema are the probable risk factors for its recurrence. Erysipelas most frequently occurs in the lower extremities, and its onset depends on the season. Therefore, while actively treating erysipelas, doctors should also understand the possible risk factors affecting erysipelas recurrence in order to prevent it.

\section{Abbreviations \\ NRE: Nonrecurrent erysipelas group; RE: Recurrent erysipelas group; CRP: C- reactive protein; WBC: White blood cell count}

\section{Acknowledgements \\ We would like to thank Prof. Xiaolin Li of Shanghai Jiao Tong University Affiliated Sixth People's Hospital for Orthopaedic Surgery, for his help.}

\section{Declarations}

Not applicable.

\section{Authors' contributions}

AL designed the study, collected, analyzed data and wrote the paper; NW and LZG collected, analyzed data; HYX and WFL performed the data evaluation. All authors read and approved the final manuscript.

\section{Funding}

This study does not have any funding to support.

\section{Availability of data and materials}

The datasets generated and analyzed during the current study are not publicly available since it contains personal information, butare available from the corresponding author on reasonable request.

\section{Ethics approval and consent to participate}

This prospective study was formally approved by the ethics committee of the First Affiliated Hospital of Shandong First Medical University (Ethic approval No. 20140020). The signed informed consent was obtained from all participants before investigation.

\section{Consent for publication}

Not applicable.

\section{Competing interests}

The authors declare that they have no competing interests.

\section{Author details}

${ }^{1}$ Department of Orthopaedic Surgery, Shanghai Jiao Tong University Affiliated Sixth People's Hospital, 600 Yishan Road, Shanghai 200233, China. 2Department of Dermatology, The First Affiliated Hospital of Shandong First Medical University, Shandong Provincial Qianfoshan Hospital, 16766 Jingshi Road, Jinan 250014, China. ${ }^{3}$ Department of Dermatology, The Second Affiliated Hospital of Shandong First Medical University, 706 Taishan Street, Tai'an 271000, China. ${ }^{4}$ Department of Surgery, Shandong Chest Hospital, 46 Lishan Road, Jinan 250013, China. 
Received: 24 April 2020 Accepted: 15 December 2020

Published online: 07 January 2021

\section{References}

1. Brishkoska-Boshkovski V, Kondova-Topuzovska I, Damevska K, Petrov A. Comorbidities as risk factors for acute and recurrent erysipelas. Open Access Maced J Med Sci. 2019;7:937-42.

2. Sunderkötter C, Becker K, Eckmann C, Graninger W, Kujath P, Schöfer H. S2k guidelines for skin and soft tissue infections excerpts from the $\$ 2 \mathrm{~K}$ guidelines for "calculated initial parenteral treatment of bacterial infections in adults - update 2018". J Dtsch Dermatol Ges. 2019:17:345-69.

3. Sunderkötter C, Becker K, Eckmann C, Graninger W, Kujath P, Schöfer H. Calculated initial parenteral treatment of bacterial infections: Skin and soft tissue infections. GMS Infect Dis. 2020;8:Doc11 eCollection 2020.

4. Pavlotsky F, Amrani S, Trau H. Recurrent erysipelas: risk factors. J Dtsch Dermatol Ges. 2004;2:89-95.

5. Jia W. Obesity in China: its characteristics, diagnostic criteria, and implications. Front Med. 2015;9:129-33.

6. McNamara DR, Tleyjeh IM, Berbari EF, Lahr BD, Martinez J, Mirzoyev SA, Baddour LM. A predictive model of recurrent lower extremity cellulitis in a population-based cohort. Arch Intern Med. 2007;167:709-15.

7. Jendoubi F, Rohde M, Prinz JC. Intracellular streptococcal uptake and persistence: a potential cause of erysipelas recurrence. Front Med. 2019;6:6

8. Mokni M, Dupuy A, Denguezli M, Dhaoui R, Bouassida S, Amri M, Fenniche S, Zeglaoui F, Doss N, Nouira R, Ben Osman-Dhahri A, Zili J, Mokhtar I, Kamoun MR, Zahaf A, Chosidow O. Risk factors for erysipelas of the leg in Tunisia: a multicenter case-controlstudy. Dermatology. 2006;212:108-12.

9. Wojas-Pelc A, Alekseenko A, Jaworek AK. Erysipelas--course of disease, recurrence, complications; a 10 years retrospective study. Przegl Epidemiol. 2007:61:457-64 Article in Polish.

10. Inghammar M, Rasmussen M, Linder A. Recurrent erysipelas--risk factors and clinical presentation. BMC Infect Dis. 2014;14:270.

11. Ezawa M, Sasaki H, Yamada K, Takano H, Iwasaka T, Nakao Y, Yokochi T, Okamoto A. Long term outcomes from lymphatic venous anastomosis after total hysterectomy to prevent postoperative lymphedema in lower limb. BMC Surg. 2019;19:177.

12. Karakonstantis S. Is coverage of S. aureus necessary in cellulitis/erysipelas? A literature review. Infection. 2019. https://doi.org/10.1007/s15010-019-01382-7 [published online ahead of print, 2019 Dec 16].

13. Rob F, Hercogová J. Benzathine penicillin G once-every-3-week prophylaxis for recurrent erysipelas a retrospective study of 132 patients. J Dermatolog Treat. 2018:29:39-43.

14. Zhu H, Han SY, Li XG, Zhou XG, Zhang QF. DNA damage in peripheral blood lymphocytes of ovarian cancer patients after radiotherapy. Eur J Gynaecol Oncol. 2013;34:450-2

15. Celenay ST, Ucurum SG, Kaya DO. Comparison of spinal alignment and mobility in women with and without post modified radical mastectomy unilateral lymphoedema. Clin Breast Cancer. 2019;20(19):30717 [Epub ahead of print].

16. Sundaresan S, Migden MR, Silapunt S. Stasis dermatitis: pathophysiology, evaluation, and management. Am J Clin Dermatol. 2017;18:383-90.

17. Brennecke S, Hartmann M, Schöfer H, Rasokat H, Tschachler E, Brockmeyer $\mathrm{NH}$. Treatment of erysipelas in Germany and Austria--results of a survey in German and Austrian dermatological clinics. J Dtsch Dermatol Ges. 2005;3: 263-70 Article in German.

18. Karppelin M, Siljander T, Vuopio-Varkila J, Kere J, Huhtala H, Vuento R, Jussila $T$, Syrjänen J. Factors predisposing to acute and recurrent bacterial nonnecrotizing cellulitisin hospitalized patients: a prospective case-control study. Clin Microbiol Infect. 2010;16:729-34.

19. Lazzarini L, Conti E, Tositti G, de Lalla F. Erysipelas and cellulitis: clinical and microbiological spectrum in an Italian tertiary care hospital. J Inf Secur. 2005:51:383-9.

20. Drerup C, Eveslage M, Sunderkoetter C, Ehrchen J. Diagnostic value of laboratory parameters for the discrimination between erysipelas and limited cellulitis. J Dtsch Dermatol Ges. 2020. https://doi.org/10.1111/ddg.14252 Online ahead of print.

\section{Publisher's Note}

Springer Nature remains neutral with regard to jurisdictional claims in published maps and institutional affiliations.

\section{Ready to submit your research? Choose BMC and benefit from:}

- fast, convenient online submission

- thorough peer review by experienced researchers in your field

- rapid publication on acceptance

- support for research data, including large and complex data types

- gold Open Access which fosters wider collaboration and increased citations

- maximum visibility for your research: over $100 \mathrm{M}$ website views per year

At $\mathrm{BMC}$, research is always in progress.

Learn more biomedcentral.com/submissions 